\title{
MicroRNA-214-5p protects against myocardial ischemia reperfusion injury through targeting the FAS ligand
}

Yuan $\mathrm{Lu}^{1}$, Jue Xi $\mathrm{X}^{2}$, Yao Zhang ${ }^{1}$, Chenzong $\mathrm{Li}^{1}$, Wensu Chen ${ }^{1}$, Xiaoqin $\mathrm{Hu}^{1}$, Min Zhang ${ }^{1}$, Fengyun Zhang ${ }^{1}$, Hui Wei ${ }^{1}$, Zhi Li ${ }^{1}$, Zhirong Wang ${ }^{1}$

\author{
1Department of Cardiology, Affiliated Hospital of Xuzhou Medical University, Xuzhou, \\ China \\ ${ }^{2}$ Department of Endocrinology, Affiliated Hospital of Xuzhou Medical University, \\ Xuzhou, China
}

Submitted: 12 November 2018

Accepted: 16 April 2019

Arch Med Sci 2020; 16 (5): 1119-1129

DOI: https://doi.org/10.5114/aoms.2019.85405

Copyright $\odot 2019$ Termedia \& Banach

\section{Abstract}

Introduction: MicroRNAs (miRNAs) are considered as crucial modulators in myocardial ischemia and reperfusion (I/R) injury. The present study aimed to investigate the expression and biological functions of miR-214-5p via targeting Fas ligand (FASLG) in I/R injury.

Material and methods: Lactate dehydrogenase, casein kinase, malondialdehyde assay, reactive oxygen species (ROS) detection and cell apoptosis analysis measured cell damage and cell apoptosis in H9c2 cells under hypox$\mathrm{ia} /$ reperfusion $(\mathrm{H} / \mathrm{R})$ treatment. Bioinformatics and dual luciferase reporter assays demonstrated the molecular mechanism of miR-214-5p in cardiac cells. 2,3,5-Triphenyltetrazolium chloride (TTC) staining, hematoxylin-eosin (HE) staining and adenovirus injection were performed in I/R treated mice. Results: The expression of miR-214-5p was decreased in H/R injured H9c2 cells compared with control cells $(p<0.001)$. Overexpression of miR-214-5p reduced cell damage and apoptosis in $\mathrm{H} 9 \mathrm{c} 2$ cells under H/R treatment ( $p<$ 0.001). Further study revealed that FASLG was a target of miR-214-5p. Enhanced expression of FASLG attenuated the protective function of miR-214$5 p$ in $H 9 c 2$ cells subjected to H/R injury $(P<0.001)$. Moreover, the elevated expression of miR-214-5p by adenovirus injection protected cardiac cells from I/R injury in mice ( $n=6 /$ per group).

Conclusions: We found that miR-214-5p exerted a protective role in I/R injured cardiac cells by direct targeting FASLG in vitro and in vivo.

Key words: ischemic heart diseases, miR-214-5p, FASLG, cell apoptosis.

\section{Introduction}

Ischemic heart disease (IHD) is a complex disease commonly caused by stenosis or occlusion of the coronary artery [1]. Myocardial ischemia/ reperfusion injury (I/R) is mainly related to re-opening after acute closure of the infarct-related artery, which results in cardiomyocyte apoptosis and necrosis. This is related to acute myocardial infarction as a specific manifestation of IHD or coronary artery disease [1-3]. However, the potential mechanism of I/R diseases still remains largely unknown.

miRNAs are a class of short non-coding RNAs that post-transcriptionally suppress the expression of the target gene by binding to its $3^{\prime}$ untranslated region (3'-UTR) [4]. miR-214 is a component of the dynamin-3

\author{
Corresponding author: \\ Dr. Zhirong Wang \\ Department \\ of Cardiology \\ Affiliated Hospital \\ of Xuzhou Medical \\ University \\ 99 Huaihai West Road \\ Quanshan District \\ Xuzhou, China \\ Phone: +86 0516-85806230 \\ E-mail: \\ Wangzhirongwzrong@163.
}

com 
gene, which is located on the chromosomal region $1 q 24.3$ [5]. Recently, several studies have reported that microRNA-124 (miR-124) participates in modulating several cardiovascular diseases including myocardial infarction, cardiac hypertrophy, pulmonary arterial hypertension and coronary artery disease [6]. miR-214 was reported to be a protector in I/R induced cardiac cell injury via inhibition of PTEN and Bim expression [7, 8]. However, the more detailed potential molecular mechanism of miR-214 in myocardial I/R needs to be further explored.

FAS ligand (FASLG), encoded by the FASLG gene, belongs to the tumor necrosis factor (TNF) superfamily [9]. When receiving a death stimulus, the binding of FASLG to FAS causes the formation of a death-inducing signaling complex (DISC) via recruitment of Fas associated via death domain (FADD) and caspase-8, which subsequently promotes cytochrome $c$ release by activating B-cell lymphoma 2 (Bcl-2) family protein-Bax but inhibiting $\mathrm{Bcl}-2$ activity, then activates caspase 9 and 3 and ultimately leads to cell death [10-13]. The FAS/FASLG induced apoptotic pathway has been reported to be associated with promoting cardiac cell apoptosis in I/R animals [14]; however, the upstream modulators of FASLG such as miRNA in cardiac I/R are still under investigation.

The present study aimed to investigate the biological function and underlying molecular mechanism of miR-214-5p in the progression of IHD.

\section{Material and methods}

\section{Cell culture and transfection}

Cardiomyocytes derived from rat embryonic ventricle ( $\mathrm{H} 9 \mathrm{c} 2$ cells) were obtained from American Type Culture Collection (ATCC, Manassas, USA). H9c2 cells were maintained in Dulbecco's modified Eagle's medium (DMEM, Gibco, Carlsbad, USA) supplemented with $10 \%$ fetal bovine serum (FBS, Gibco) and $100 \mathrm{U} / \mathrm{ml}$ penicillin/streptomycin (Gibco) at $37^{\circ} \mathrm{C}$ in a humidified with $5 \%$ $\mathrm{CO}_{2}$ atmosphere.

For transfection, cells were seeded into a plate at appropriated density. miR-214-5p-mimic, miR214-5p-inhibitor and FASLG pcDNA plasmid were acquired from GenePharma (Shanghai, China). Cells were transfected with miRNAs at a final concentration of $40 \mathrm{nM}$ or plasmid using Lipofectamine 2000 Reagent (Invitrogen, Carlsbad, USA) following the manufacturer's protocol.

\section{Hypoxia/reoxygenation (H/R) treatment in vitro}

To construct ischemia in vitro, cells were transfected with the miR-214-5p mimic or inhibitor for
48 h. Then H9c2 cells were cultured in medium with $1 \%$ FBS (low serum medium) followed by exposure to hypoxia in a chamber with $94 \% \mathrm{~N}_{2}$, $5 \% \mathrm{CO}_{2}$ and $1 \% \mathrm{O}_{2}$ for $10 \mathrm{~h}$. Then, the low serum medium was removed and the cells were cultured again with $10 \%$ FBS-containing DMEM medium in a normoxic environment for reoxygenation for $2.5 \mathrm{~h}$. After that, the cells were collected to perform experiments. Cells were cultured in medium with $1 \%$ FBS for $10 \mathrm{~h}$ and then re-cultured in $10 \%$ FBS for $2.5 \mathrm{~h}$ in a normoxic environment to serve as a control.

\section{Measurement of lactate dehydrogenase (LDH) and casein kinase (CK) levels}

After I/R treatment, serum from the ventricle was collected for detection of LDH and CK activity. The LDH release and the CK activity (U/I) were determined by an LDH assay kit (Solarbio, Beijing, China) and CK assay kit (Solarbio), respectively.

\section{Malondialdehyde (MDA) assay}

The expression of MDA was detected with a micro MDA assay kit (Solarbio). Briefly, cells were incubated with thiobarbituric acid (TBA, 2.8\% $\mathrm{w} / \mathrm{v}$ ) at $95^{\circ} \mathrm{C}$ for $60 \mathrm{~min}$ and then centrifuged at $4^{\circ} \mathrm{C}, 2,500 \times \mathrm{g}$ for $10 \mathrm{~min}$. The reactive complexes were then detected using a NanoDrop One/Onec spectrophotometer at $532 \mathrm{~nm}$ (Thermo Fisher Scientific).

\section{Reactive oxygen species (ROS) detection assay}

Cells were digested with trypsin at $37^{\circ} \mathrm{C}$ for $30 \mathrm{~min}$ and then incubated with $2^{\prime}, 7^{\prime}$-dichlorofluo-rescin diacetate (DCFH-DA) at a final concentration of $10 \mu \mathrm{M}$ for $1 \mathrm{~h}$ at $37^{\circ} \mathrm{C}$. Finally, the images were acquired by fluorescence microscope (Olympus, Tokyo, Japan) and the production of ROS was assessed by the static cytometry software ScanR version 2.03.2 (Olympus).

\section{Cell apoptosis analysis}

The cell apoptosis was determined by an Annexin V FITC/Propidium lodide (PI) apoptosis kit (BD Pharmingen, San Jose, USA) using the flow cytometer (Becton-Dickinson, Franklin Lakes, USA). After incubation with Annexin V-FITC for 5 min in the dark, cells were treated with PI and RNase $A$ at the same final concentration of $10 \mathrm{mg} / \mathrm{ml}$ for $30 \mathrm{~min}$ at $4^{\circ} \mathrm{C}$. Then the cells were immediately checked by flow cytometer and analyzed by Cell-Quest software (Becton-Dickinson). The right upper quadrant and the right lower quadrant indicated the apoptotic cells labeled with Annexin V-FITC. 


\section{Western blot}

Proteins were extracted using a RIPA buffer (Beyotime) and quantified using a BCA kit (Beyotime). $30 \mu \mathrm{g}$ of total proteins from each sample were separated with SDS-PAGE gel and then transferred onto polyvinylidene fluoride (PVDF) membrane (EMD Millipore, Billerica, USA). After being blocked in 5\% skim milk, membranes were incubated with primary antibodies against cleaved-caspase 3 (Abcam, Cambridge, UK; dilution rates of $1: 500$ ), cleaved-caspase 9 (Abcam, dilution rates of 1 : 500), Bax (Abcam, dilution rates of 1 : 1000), Bcl-2 (Abcam, dilution rates of $1: 500$ ) or FASLG (Abcam, dilution rates of $1: 300$ ) overnight at $4^{\circ} \mathrm{C}$. The next day, membranes were incubated with goat anti-rabbit (Abcam, dilution rates of 1 : 2000) or goat anti-mouse (Abcam, dilution rates of 1 : 2000) horseradish peroxidase (HRP)-linked secondary antibody for $1 \mathrm{~h}$ at room temperature. Finally, images of bands were detected using an enhanced chemiluminescent detection kit (Thermo Fisher Scientific) and quantified using Image Lab software 3.0 (Bio-Rad, Hercules, USA).

\section{Quantitative real-time PCR (qRT-PCR)}

Total RNAs were isolated using Trizol reagent (Invitrogen) and then reverse transcribed into cDNA by a reverse transcription reagent kit ( $\mathrm{Ta}$ kara, Dalian, China). The relative expression of miRNA was performed using SYBR Green PCR Master Mix (Takara) and detected using the BioRad Real-Time PCR instrument (Bio-Rad). The relative level of miRNA was normalized with $\mathrm{U} 6$ and quantitative analysis was calculated using the $2^{-\Delta \Delta \mathrm{ct}}$ method. The primer sequences are shown in Table I.

\section{Dual luciferase activity assay}

The fragments of the $3^{\prime}$-UTR of the FASLG gene with a type or mutant FASLG were obtained from the Beijing Genomics Institute (BGI, Beijing, China) and then cloned into the pGL3-vector (Promega, Madison, USA). The H9c2 cells were co-transfected with $50 \mathrm{ng}$ of luciferase vectors, $10 \mathrm{ng}$ of pRL-
TK vectors and $50 \mathrm{nM}$ miR-214-5p mimics or its control mimics (NC mimics) using Lipofectamine 2000 reagent (Invitrogen). After transfection for $48 \mathrm{~h}$, the cells were lysed and then the luciferase activities were determined using the Dual Luciferase Reporter Assay System (Promega). The data were evaluated by the ratio of firefly fluorescent value to Renilla fluorescence value.

\section{Ischemia/reperfusion (I/R) in vivo}

The male C57BL/6 mice ( 8 weeks, 20-25 g) were purchased from Shanghai Laboratory Animal Center (Shanghai, China). They were maintained at a standard temperature of $22 \pm 2^{\circ} \mathrm{C}$ and a programmed $12 \mathrm{~h}$ light/dark cycle with free access to food and water. All animal experimental procedures were approved by the Animal Research Ethics Committee of the Affiliated Hospital of Xuzhou Medical University in accordance with the National Institutes of Health Guide for the Care and Use of Laboratory Animals.

All mice were anesthetized with $2 \%$ sodium phenobarbital (50 mg/kg) via intraperitoneal administration and maintained with a rectal temperature of $37^{\circ} \mathrm{C}$ using a homeothermic table. After fixing for endotracheal intubation using a respirator, a longitudinal incision was made from the third to fourth ribs. The myocardial ischemia was performed by ligating the proximal left anterior descending artery (LAD) using a 5-0 polypropylene suture for 30 min followed by $2 \mathrm{~h}$ of reperfusion. Next, mice were re-anesthetized and heart tissues were removed for the further study. In the Sham groups, the same operation was performed but the LAD was not ligated.

\section{Ad-miR-214-5p treatment animals}

The miR-214-5p overexpression adenovirus (Ad-miR-214-5p) was obtained from the Shanghai Genechem Co. (Shanghai, China). The mice were treated with Ad-miR-214-5p 5 days before the I/R operation. After anesthetization, the chest was opened and Ad-miR-214-5p was administered into 8 different sites of the left ventricular. The mouse chest was closed and the mice were allowed to recover in their cage.

Table I. Primer sequences used in qRT-PCR

\begin{tabular}{|lcc|}
\hline Name & Primer & Sequence (5'-3') \\
\hline U6 & Forward & 5'-CTCGCTTCGGCAGCACA-3' \\
\cline { 2 - 3 } & Reverse & 5'-AACGCTTCACGAATTTGCG-3' \\
\hline miR-214-5p & RT primer & 5'-CTCAACTGGTGTCGTGGAGTCGGCAATTCA GTTGAGGCACAGC-3' \\
\cline { 2 - 3 } & Forward & 5'-ACACTCCAGCTGGGTGCCTGTCTACACT-3' \\
\cline { 2 - 3 } & Reverse & 5'-CCAGTGCAGGGTCCGAGGT-3' \\
\hline
\end{tabular}




\section{2,3,5-Triphenyltetrazolium chloride (TTC) assay}

TTC assay was carried out to determine the infarct size of heart tissues. In brief, after refrigeration at $-20^{\circ} \mathrm{C}$ for $30 \mathrm{~min}$, the left ventricle was acquired and transversely cut into $2 \mathrm{~mm}$ thick slices and stained with $1 \%$ tri-phenyltetrazolium chloride (TTC, sigma-Aldrich) at $37^{\circ} \mathrm{C}$ for $20 \mathrm{~min}$. Then the slices were photographed, and the area at risk (AAR) and infarct size were analyzed and calculated. The infarct size was expressed as a percentage of the AAR.

\section{Hematoxylin-eosin (HE) staining}

The hearts were harvested, fixed in $4 \%$ paraformaldehyde, and embedded in paraffin. Next, the tissue was cut into $5 \mu \mathrm{m}$ sections. After hydration and xylene dewaxing, the sections were stained with hematoxylin and eosin (Solarbio). Finally, the sections were photographed under a light microscope (Leica, Wetzlar, Germany).

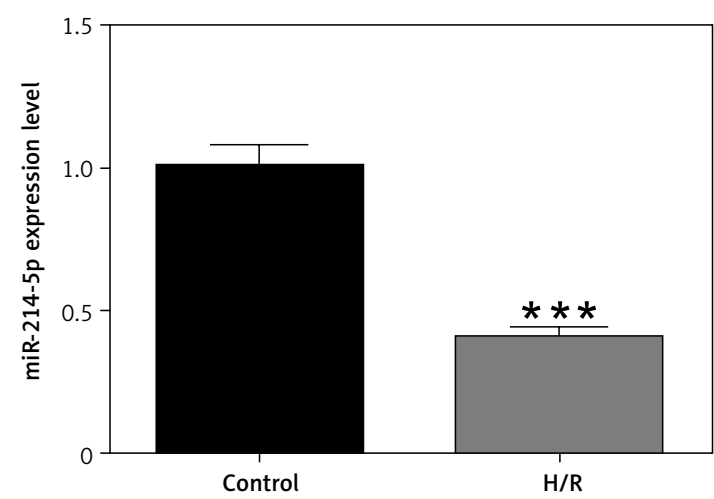

Figure 1. The expression of miR-214-5p was decreased in $\mathrm{H} 9 \mathrm{c} 2$ cells under $H / R$ conditions. The expression of miR-214-5p in H9c2 cells under H/R treatment was detected by qRT-PCR

Data are presented as mean $\pm S D$. ${ }^{* *} P<0.001$.

\section{Statistics analysis}

All data were expressed as mean \pm standard deviation (SD). Statistical analysis was performed using SPSS 16.0 (SPSS, Chicago, USA). Statistical differences among groups were assessed by oneway analysis of variance (ANOVA) with Tukey's post hoc test. A value of $p<0.05$ was considered as statistically significant. All experiments were performed at least 3 separate times with similar results.

\section{Results}

\section{miR-214-5p was down-regulated in $\mathrm{H} 9 \mathrm{c} 2$ cells after $H / R$ injury}

H9c2 cells were exposed to hypoxia for $10 \mathrm{~h}$ followed by reoxygenation for $2.5 \mathrm{~h}$, and then the expression of miR-214-5p was detected by qRT$P C R$. As shown in Figure 1, the expression of miR214-5p was dramatically decreased in H9c2 cells after simulated $\mathrm{H} / \mathrm{R}$ injury compared with control cells $(p<0.001$, Figure 1$)$. These data suggested that miR-214-5p was down-regulated under ischemic conditions in vitro.

\section{miR-214-5p attenuated cardiac cell deficit insult to $H / R$ exposure}

To explore the function of miR-214-5p expression in I/R injury, up- or down- regulation of miR214-5p expression by transfection with miR-214 mimic or inhibitor was performed in $\mathrm{H} 9 \mathrm{c} 2$ cells before exposure to $H / R$ impairment. As shown in Figure $2 \mathrm{~A}$, the expression level of miR-214-5p was markedly increased by miR-214-5p mimic while it was decreased upon miR-214-5p inhibitor treatment in $\mathrm{H} / \mathrm{R}$ treated $\mathrm{H} 9 \mathrm{c} 2$ cells compared with the group with only $\mathrm{H} / \mathrm{R}$ treatment $(p<0.001)$. As a critical sign of cell deficit, the $\mathrm{LDH}$ release from the H9c2 cells was detected. The result suggest-
A

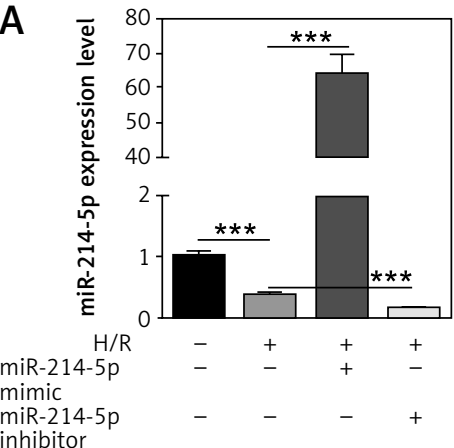

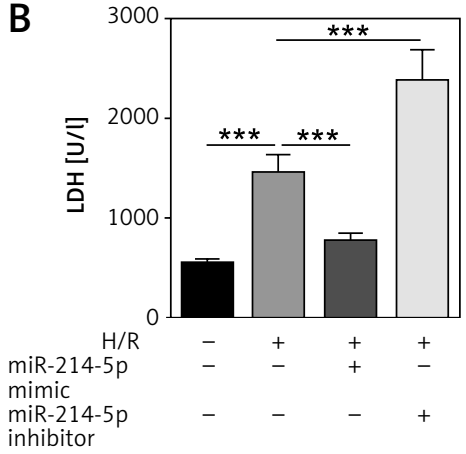

Figure 2. miR-214-5p attenuated H/R treatment induced cardiomyocyte injury. H9c2 cell lines were transfected with miR-214-5p mimic or inhibitor for $24 \mathrm{~h}$ followed by $\mathrm{H} / \mathrm{R}$ treatment. A - The effect of miR-214-5p mimic or inhibitor on the expression level of miR-214-5p was detected by qRT-PCR in H9c2 cells under H/R conditions. B - The effect of up- or down-regulation of miR-214-5p expression on the LDH activity was measured by LDH assay in cardiac cells upon H/R treatment conditions. $\mathrm{C}$ - The role of overexpression or downregulation of miR-214-5p on the MDA activity was determined by MDA detection assay

Data are presented as mean $\pm S D$. ${ }^{* * *} P<0.001$. 
Control

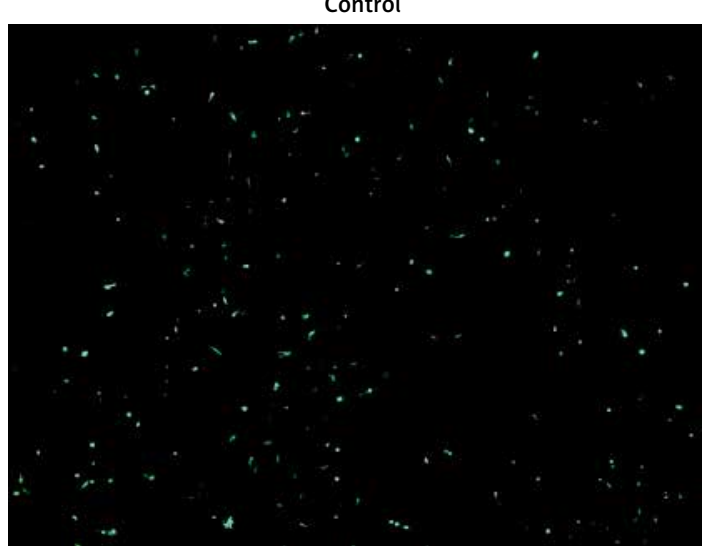

$H / R+$ miR-214-5p mimic
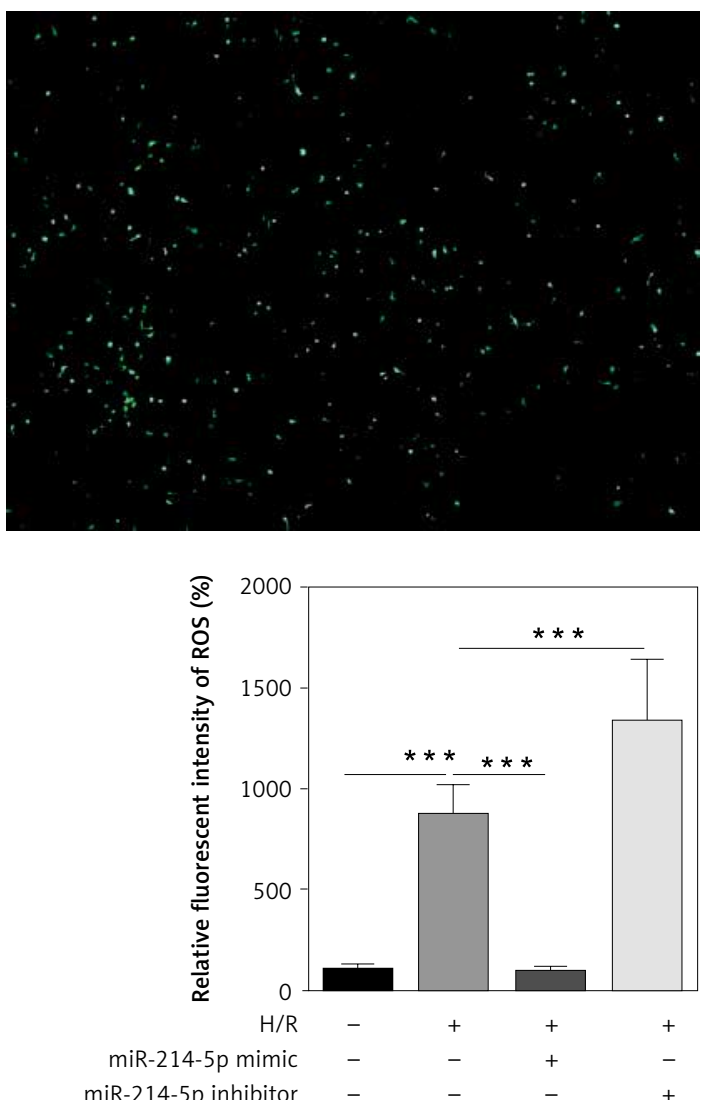

ed that the LDH activity was enhanced after exposure to $H / R$ injury, and this enhancement was reversed by transfection with miR-214-5p mimic while being further elevated by treatment with miR-214-5p inhibitor ( $p<0.001$, Figure 2 B). As a vital indicator of lipid peroxidation, MDA was further measured using the MDA assay kit. The result showed that the increased expression levels of MDA were reduced by miR-214-5p mimic but further elevated by miR-214-5p inhibitor in H/R treated H9c2 cells ( $p<0.001$, Figure 2 C). In addition, ROS are a major product in response to hypoxia-induced oxidative stress. As shown in Figure 3 , the elevated relative fluorescent intensity of

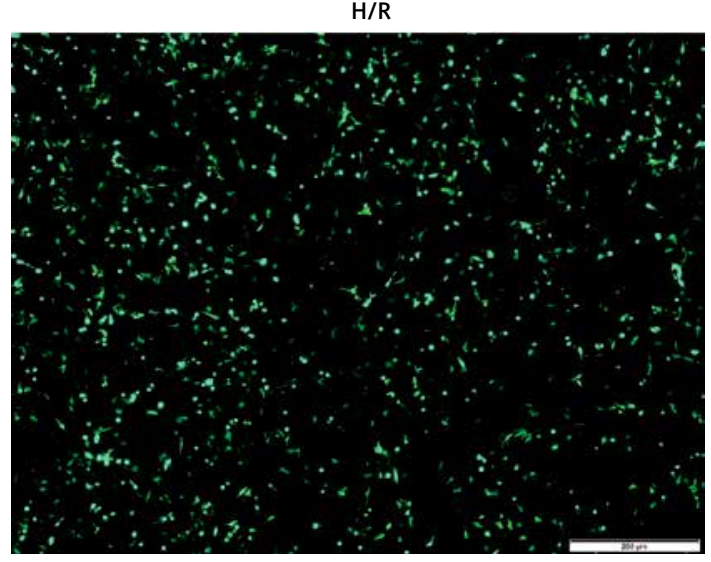

$H / R+$ miR-214-5p inhibitor

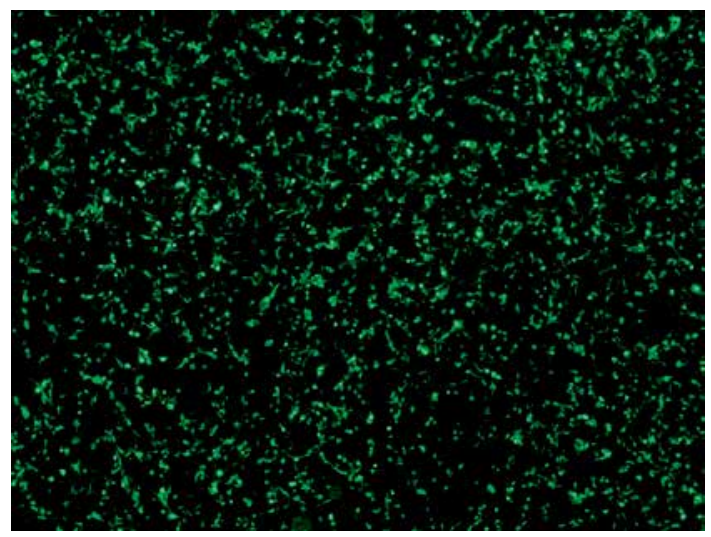

Figure 3. miR-214-5p reduced H/R-induced ROS production in cardiomyocytes. The function of elevated or reduced expression of the miR-214-5p by its mimic or inhibitor at the ROS generation level was evaluated by ROS detection assay after H9c2 cells' insult due to H/R injury

Data are presented as mean $\pm S D$. ${ }^{* * *} P<0.001$.

ROS in response to H/R injury was suppressed by miR-214-5p mimic while being further enhanced by miR-214-5p inhibitor in H9c2 cells ( $p<0.001$, Figure 3 ). Thus, the data confirmed that overexpression of miR-214-5p ameliorated H/R induced cardiac cell damage and ROS production in vitro.

\section{miR-214-5p suppressed H9c2 cell apoptosis subjected to $H / R$}

Further, the effect of overexpression or interference of miR-214-5p on the H/R injury induced cardiomyocyte apoptosis was detected. Flow cytometry results indicated that the cardiac cell apoptosis 
rate was notably increased by $\mathrm{H} / \mathrm{R}$ impairment, which was further elevated by miR-214-5p inhibitor but decreased by miR-214-5p mimic in H9c2 cells $(p<0.001$, Figure 4 A). Moreover, the expression levels of anti-apoptosis protein $\mathrm{Bcl}$-2, pro-apoptosis protein Bax, cleaved-caspase 3 and cleaved-caspase 9 were examined when the expression of miR-214$5 p$ in H/R injured cells was up- or down-regulated. As shown in Figure $4 \mathrm{~B}$, the decreased expression of $\mathrm{BCl}-2$ induced by $\mathrm{H} / \mathrm{R}$ treatment was up-regulated by miR-214-5p mimic but further down-regulated by miR-214-5p inhibitor $(p<0.01, p<0.001)$. In contrast, the increased expression levels of Bax, cleaved-caspase 3 and cleaved-caspase 9 induced by $H / R$ treatment were attenuated by miR-214-5p mimic but further elevated by miR-214-5p inhibitor in H9c2 cells ( $p<0.01, p<0.001$, Figure 4 B). Taken together, these data indicated that overexpression of miR-214-5p is able to inhibit cardiomyocyte apoptosis induced by $\mathrm{H} / \mathrm{R}$ treatment.

A
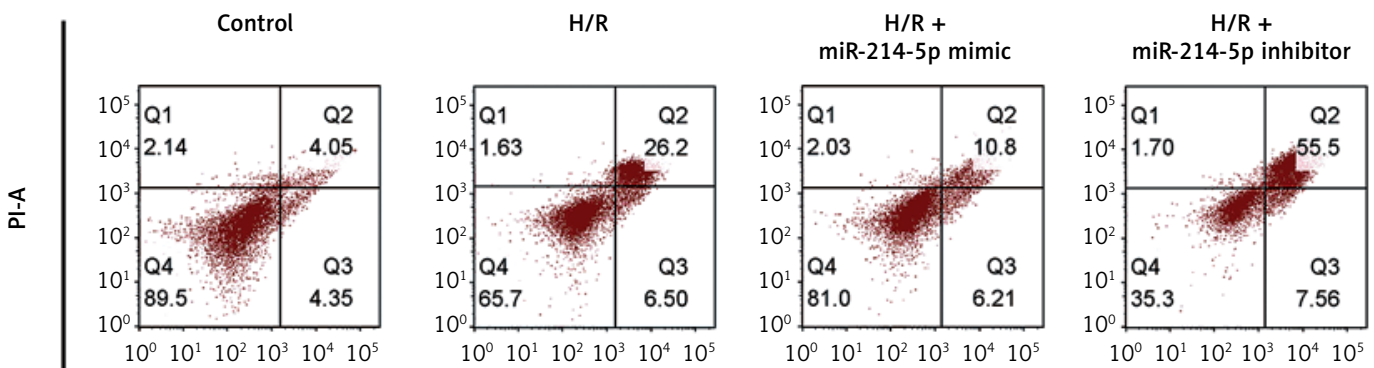

Annexin V-FITC

\section{B}
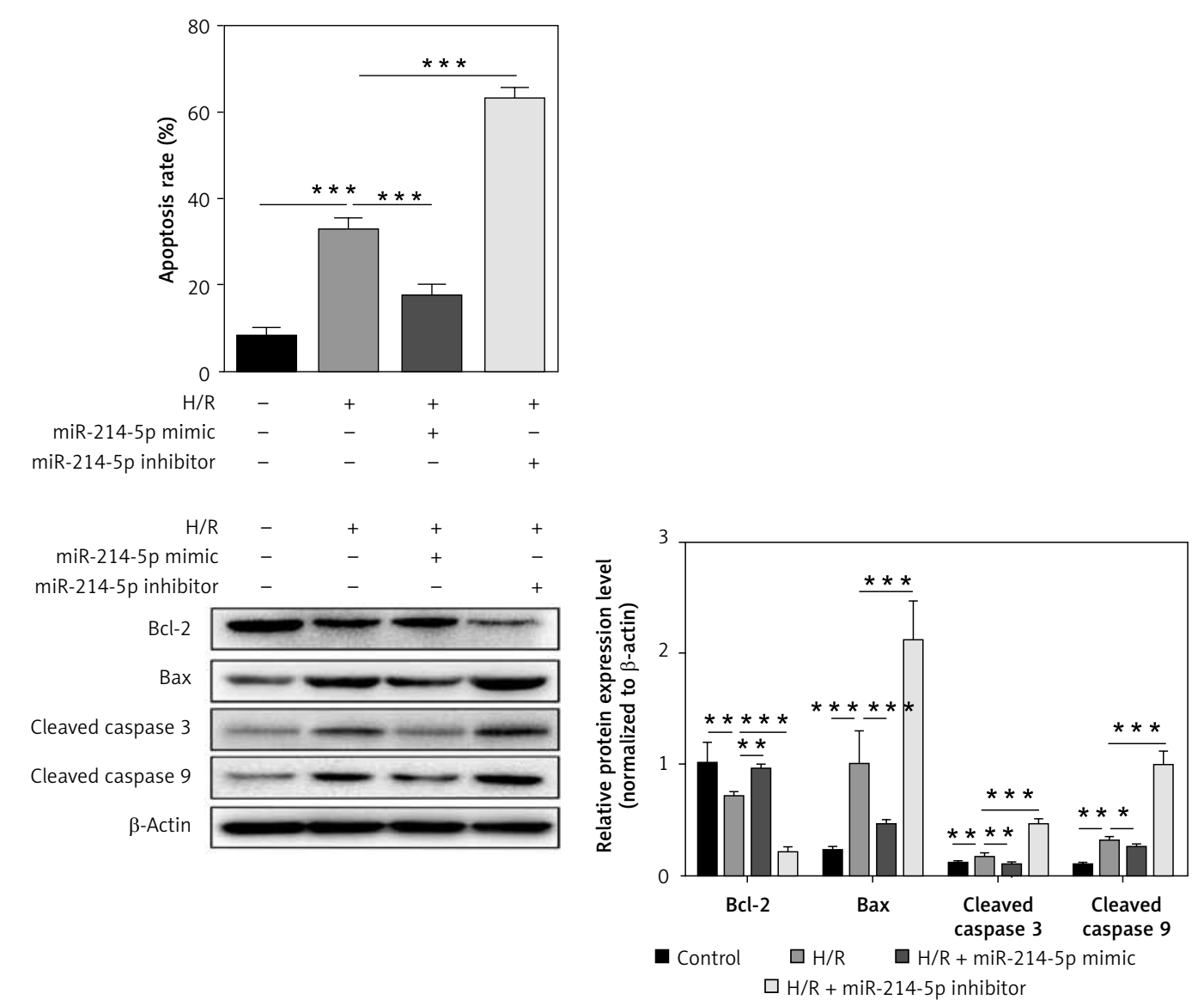

Figure 4. miR-214-5p suppressed apoptosis of cardiac cells subject to $H / R$ impairment. A - The effect of overexpression or silencing of miR-214-5p in H9c2 cells after H/R treatment on apoptosis rate of cardiac cells was tested by cell apoptosis analysis using a flow cytometer. B - The effect of enhancing or interference of miR-214-5p expression levels on the anti-apoptotic proteins Bcl-2 or pro-apoptotic proteins including Bax, cleaved-caspase 3 and cleaved-caspase 9 was determined by western blotting in $\mathrm{H} 9 \mathrm{c} 2$ cells after $\mathrm{H} / \mathrm{R}$ injury

Data are presented as mean $\pm S D ;{ }^{*} p<0.05,{ }^{* *} p<0.01,{ }^{* * *} p<0.001$. 


\section{FASLG was a target gene of miR-214-5p}

To elucidate the underlying molecular mechanism of miR-214-5p inhibiting H/R-induced cardiomyocyte injury, we used miRBase (http://www. mirbase.org/) and TargetScan online tools (http:// www.targetscan.org/vert_71/) to screen potential genes that have a binding site with miR-214-5p. FASLG was predicted as a target gene as its mRNA $3^{\prime}$-UTR region contains binding sites complementary to miR-214-5p (Figure $5 \mathrm{~A}$ ), which was further confirmed by dual luciferase report assay. We constructed FASLG 3'-UTR wild-type (FASLG 3'-UTRwt) luciferase plasmids containing the potential miR-214-5p binding sites as well as a mutated version of each site (FASLG 3'-UTR-mut) (Figure $5 \mathrm{~A}$ ). As shown in Figure $5 \mathrm{~B}$, the luciferase activity of $\mathrm{H} 9 \mathrm{c} 2$ cells transfected with FASLG 3'-UTR-wt plasmid was markedly attenuated by miR-214-5p mimic $(p<0.001)$, while the luciferase activity did not change in cells transfected with FASLG 3'-UTRmut vector $(p>0.05)$. Next, the effect of altered miR-214-5p expression on the FASLG protein level was evaluated. The elevated protein level of
FASLG upon exposure to H/R injury was inhibited by miR-214-5p mimic but increased by miR-214$5 p$ inhibitor $(p<0.001$, Figure 5 B). These data suggested that FASLG is a target of miR-214-5p and its expression was negatively regulated by miR-214-5p.

\section{Overexpression of FASLG abolished \\ the cardioprotective roles of miR-214-5p \\ in $\mathrm{H} / \mathrm{R}$ injured $\mathrm{H} 9 \mathrm{c} 2$ cells}

Based on the above data, we found that miR214-5p decreased H/R treatment-induced H9c2 cell apoptosis and negatively regulated FASLG expression. Then we evaluated the effect of exogenous up-regulated expression of FASLG by transfection with its overexpression pcDNA plasmid on the cardiomyocytes treated with miR-214-5p mimic under $\mathrm{H} / \mathrm{R}$ treatment. As shown in Figure $6 \mathrm{~A}$, miR-214$5 p$ mimic treatment decreased the LDH and MDA release level but it was enhanced by FASLG pcDNA in $\mathrm{H} / \mathrm{R}$ treated $\mathrm{H} 9 \mathrm{c} 2$ cells $(p<0.001$, Figure 6 A). Compared with transfection with miR-214-5p mimic alone, the ROS production was restored to

A

Position 18-24 of FASLG
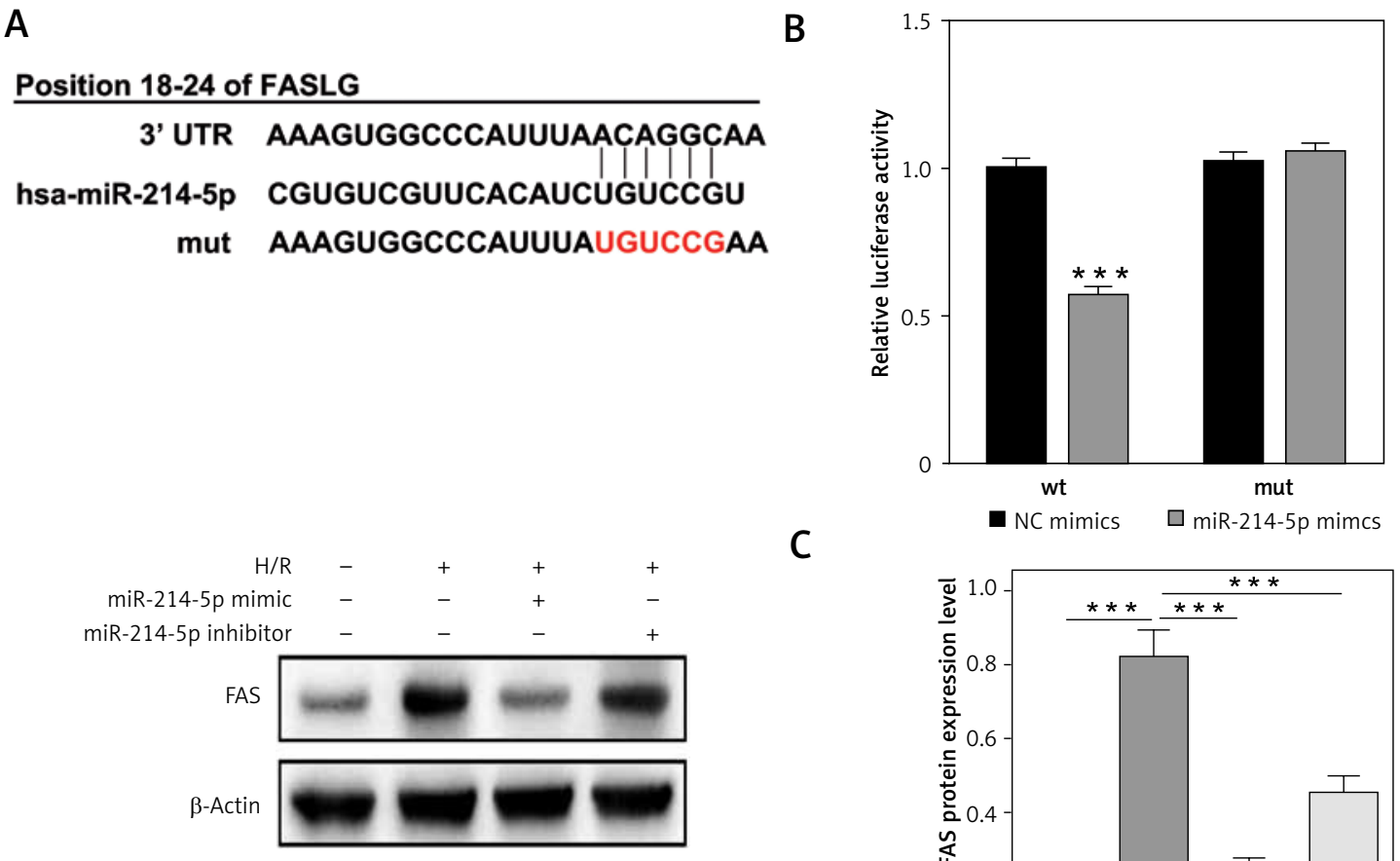

C

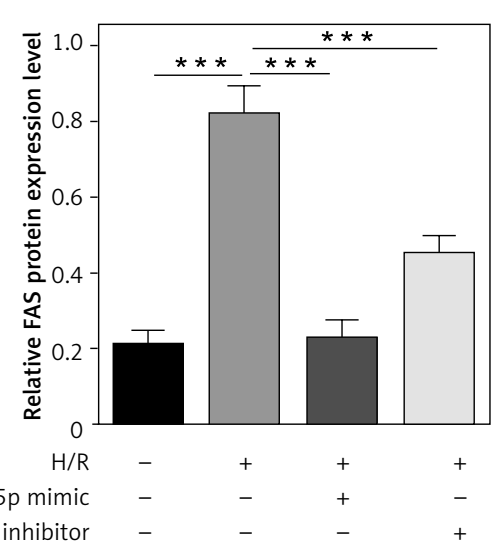

Figure 5. FASLG was targeted by miR-214-5p. A - The 3'-UTR of FASLG mRNA containing the predicted target sites for miR-214-5p (FASLG-3'-UTR-wt) or the mutated miR-214-5p binding sites in the FASLG gene (FASLG-3'-UTRmut) are shown. B - Luciferase activities of H9c2 cells co-transfected with miR-214-5p mimics or NC mimics and luciferase reporters containing FASLG 3'-UTR-wt or -mut transcript were checked by dual-luciferase reporter assays. C - The effect of up- or down-regulated expression of miR-214-5p on the protein level of FASLG was measured by western blot in H/R-treated H9c2 cells

Data are presented as mean $\pm S D$. ${ }^{* * *} P<0.001$. 
A

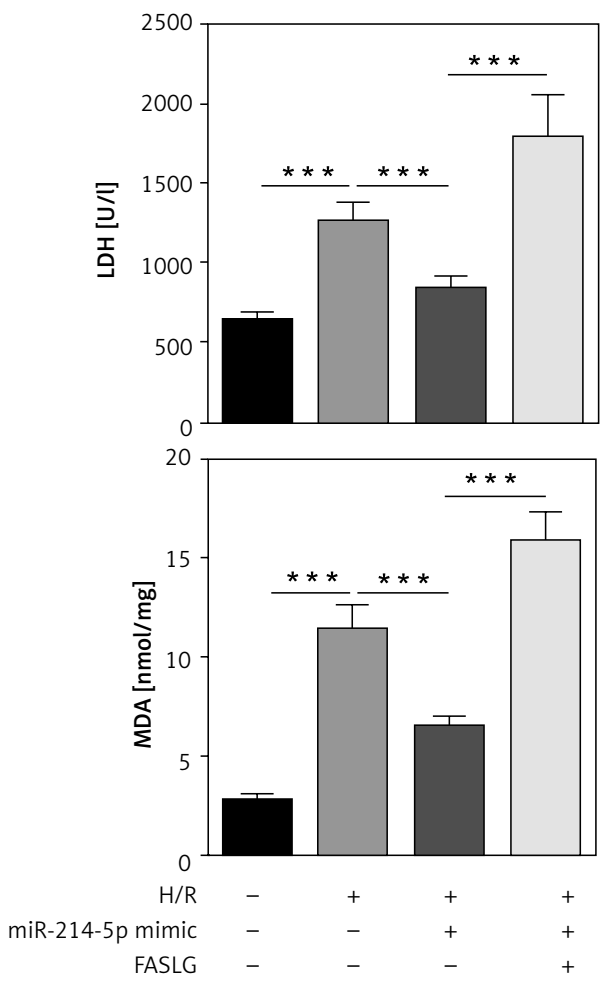

C
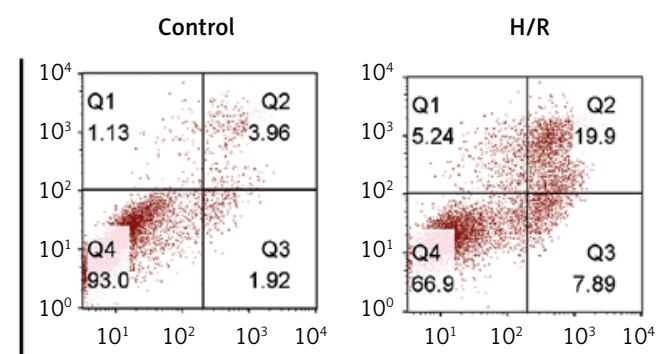

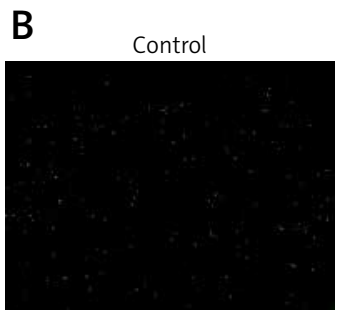

$H / R+$ miR-214-5p mimic

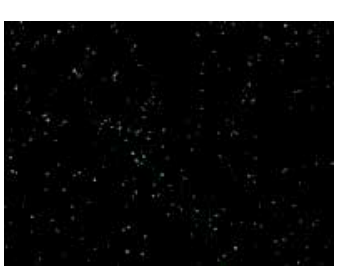

$H / R+$ miR-214-5p mimic + FASLG
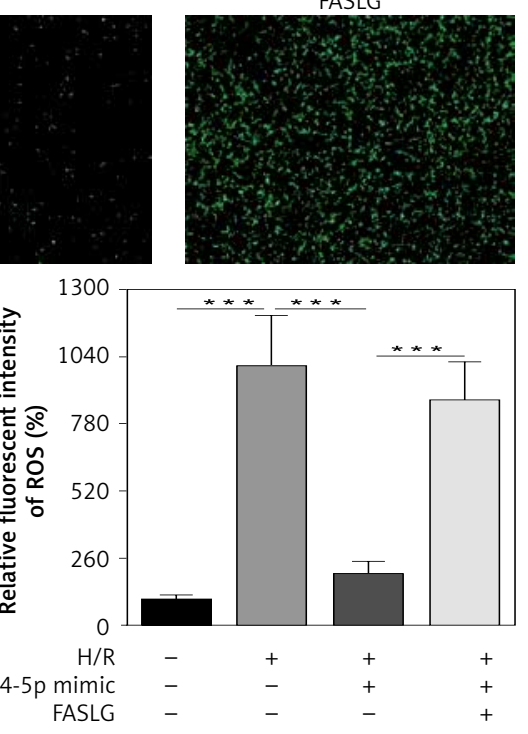

$\mathrm{H} / \mathrm{R}+$

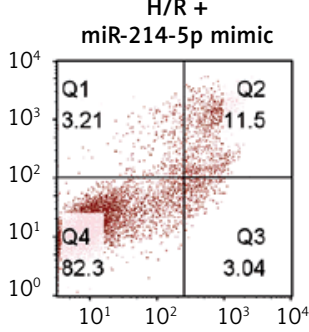

$\mathrm{H} / \mathrm{R}+$

miR-214-5p mimic + FASLG

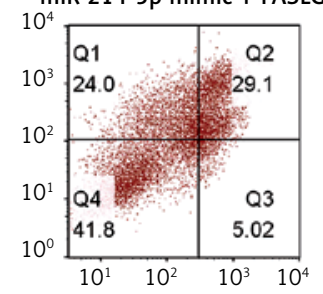

Annexin V-FITC

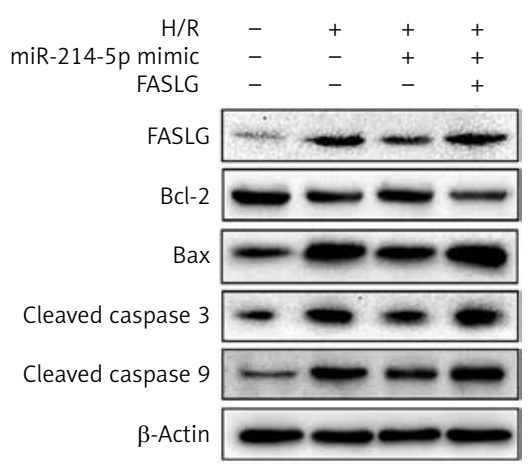

Figure 6. The protective role of miR-214-5p on the H/R injured cardiac cells was blocked by overexpression of FASLG. The H9c2 cells were co-transfected with miR-214-5p mimic along with FASLG pcDNA followed by H/R treatment. A - The influence of overexpression of miR-214-5p and up-regulation of FASLG on the LDH and MDA activities was tested by LDH and MDA assay kit in H9c2 cells under H/R treatment. B - The effects of enhanced miR-214-5p level and elevated expression of FASLG on ROS production were evaluated by ROS detection assay in $\mathrm{H} 9 \mathrm{c} 2$ cells upon $\mathrm{H} / \mathrm{R}$ exposure. C - The effects of up-regulated miR-214-5p level and enhanced FASLG expression on the apoptosis rate were determined by cell apoptosis analysis assay in $\mathrm{H} 9 \mathrm{c} 2$ cells by $\mathrm{H} / \mathrm{R}$ treatment. D - The effect of advanced expression level of miR-214-5p when co-transfected with FASLG overexpression construct in $\mathrm{H} 9 \mathrm{c} 2$ cells on the protein levels including FASLG, Bcl-2, Bax, cleaved-caspase 9 and cleavedcaspase 3 was measured by western blotting upon $H / R$ exposure Data are presented as mean $\pm S D$. ${ }^{* * *} P<0.001$. 
a high level after co-transfection of FASLG pcDNA in $\mathrm{H} / \mathrm{R}$-treated $\mathrm{H} 9 \mathrm{c} 2$ cells $(p<0.001$, Figure $6 \mathrm{~B})$. Further, the cardiac cell apoptosis rate and pro-apoptosis protein expression levels were also detected. As shown in Figure $6 \mathrm{C}$, the decreased cell apoptosis rate induced by miR-214 mimic was returned to a high level by up-regulation of FASLG in H/R treated $\mathrm{H} 9 \mathrm{c} 2$ cells $(p<0.001)$. In addition, western blot results showed that the overexpression of FASLG in cardiomyocytes after H/R injury dramatically enhanced the expression of FASLG and the pro-apoptosis factors Bax, cleaved caspase- 3 and cleaved caspase-9 $(p<0.001)$, whereas it decreased the expression of the anti-apoptosis factor $\mathrm{Bcl}-2 \mathrm{com}$ pared to the miR-214-5p treatment alone ( $p<$ 0.001 , Figure $6 \mathrm{D})$. Altogether, the roles of miR-214$5 p$ in decreasing ROS generation and cell apoptosis were abolished by overexpression of FASLG in cardiac cells under $\mathrm{H} / \mathrm{R}$ treatment.

\section{Overexpression of miR-214-5p alleviated myocardial injury induced by I/R treatment in mice}

Further, the cardioprotective function of miR214-5p was confirmed via intracoronary injection of miR-214-5p overexpression adenovirus (Ad-miR-214-5p) into I/R treated mice. After administration of Ad-miR-214-5p 5 days before I/R operation, the miR-214-5p expression was notably up-regulated compared with the control or I/R group $(p<0.001$, Figure 7 A). The enhanced expression of miR-214-5p decreased the CK and LDH activity in I/R treated mice $(p<0.001$, Figure $7 \mathrm{~B})$. Moreover, the infarct size and heart damage were markedly reduced by Ad-miR-214-5p injection in animals operated on with I/R treatment ( $p<$ 0.001 , Figure $7 \mathrm{D}$ ). As shown in Figure $7 \mathrm{E}$, the disordered arrangement of myocardial fibers and the inflammatory cell infiltration in the interstitium in I/R treated mice were ameliorated by up-regulation of miR-214-5p. Further, compared with the I/R treated group, the levels of FASLG protein and pro-apoptotic proteins including Bax, cleavedcaspase 9 and cleaved-caspase 3 were dramatically attenuated by Ad-miR-214-5p ( $p<0.01, p<$ 0.001 ) whereas the expression of anti-apoptotic protein $\mathrm{Bcl}-2$ was increased by overexpression of miR-214-5p in I/R treated mice $(p<0.001$, Figure $7 \mathrm{~F})$. Thus, overexpression of miR-214-5p reduced heart infarct size and cardiac cell apoptosis and ultimately alleviated cardiac tissue damage insult due to ischemic injury in vivo.

\section{Discussion}

The present study revealed that the expression level of miR-214-5p was decreased in H/R injured $\mathrm{H} 9 \mathrm{c} 2$ cells. Overexpression of miR-214-5p was able to reduce cell injury and cell apoptosis in $\mathrm{H} 9 \mathrm{c} 2$ cells upon H/R treatment and in mice treated with an I/R operation. Apoptosis is a major event linked with IHD [15]. Our study demonstrated that miR214 protected cardiac cells from I/R injury via targeting pro-apoptosis protein-FASLG in $\mathrm{H} 9 \mathrm{c} 2$ cells. These results suggested that miR-214-5p might be a protective factor for $\mathrm{I} / \mathrm{R}$ injury by inhibiting FASLG expression.

miR-214 is a highly conserved miRNA among species, which is associated with various physiological and pathological processes of cardiovascular diseases including IHD $[5,6]$. Overexpression of miR-214 by its lentivirus attenuated cardiomyoblasts injury, decreased myocardial infarct size and ultimately improved the cardiac function in I/R treated mouse, via activation of PI3K/Akt signaling by targeting PTEN expression and suppressing hypoxia inducible factor $1[7,16]$. Interestingly, miR-214 is considered as a target for treatment of $I / R$ diseases due to its protective roles in I/R disease. For instance, the nonselective $\beta$-adrenergic receptor antagonist carvedilol or electroacupuncture pretreatment enhanced cardiac function and inhibited cardiomyocyte damage upon simulate I/R injury via enhancing miR-214 expression $[8,17]$. In the present study, overexpression of miR-214-5p was able to reduce the cardiac cell damage and apoptosis induced by I/R injury in vivo and in vitro. Thus, our study suggested that miR-214 plays a protective role against I/R injury in IHD.

miRNA exerts its functions via regulating various target genes [4]. Hence, establishing the relationship of miR-214-5p and its target gene may contribute to understanding the molecular mechanism underlying I/R impairment. In our study, FASLG was predicted as a target gene of miR214-5p, and further study confirmed that FASLG overexpression attenuated the cardioprotective role of miR-214-5p in H/R injured cardiac cells. It is well recognized that cardiomyocyte apoptosis is a fundamental feature in response to I/R injury [18]. FASLG binding with its receptor FAS could trigger extrinsic and intrinsic apoptosis pathways that were mediated by a series of apoptosis related proteins when the death signal was stimulated [10-13]. The mRNA and protein levels of FAS/ FASLG were notably elevated in animals operated on by the coronary artery ligation constructed I/R model [14]. Moreover, FAS/FASLG was closely associated with coronary disease and is considered as a risk factor in CAD patients [19, 20]. FASLG/FAS and its downstream factors such as Bax, cleavedcaspase 9 or 3 are the treatment targets for I/R disease cure [21-23]. For instance, Toll like receptors (TLRs), hydrogen sulphide (H2S) tadalafil or luteolin-7-O-glucoside display their anti-apoptotic activity against I/R via down-regulation of FASLG/FAS and its downstream signalling factor [21-23]. Con- 

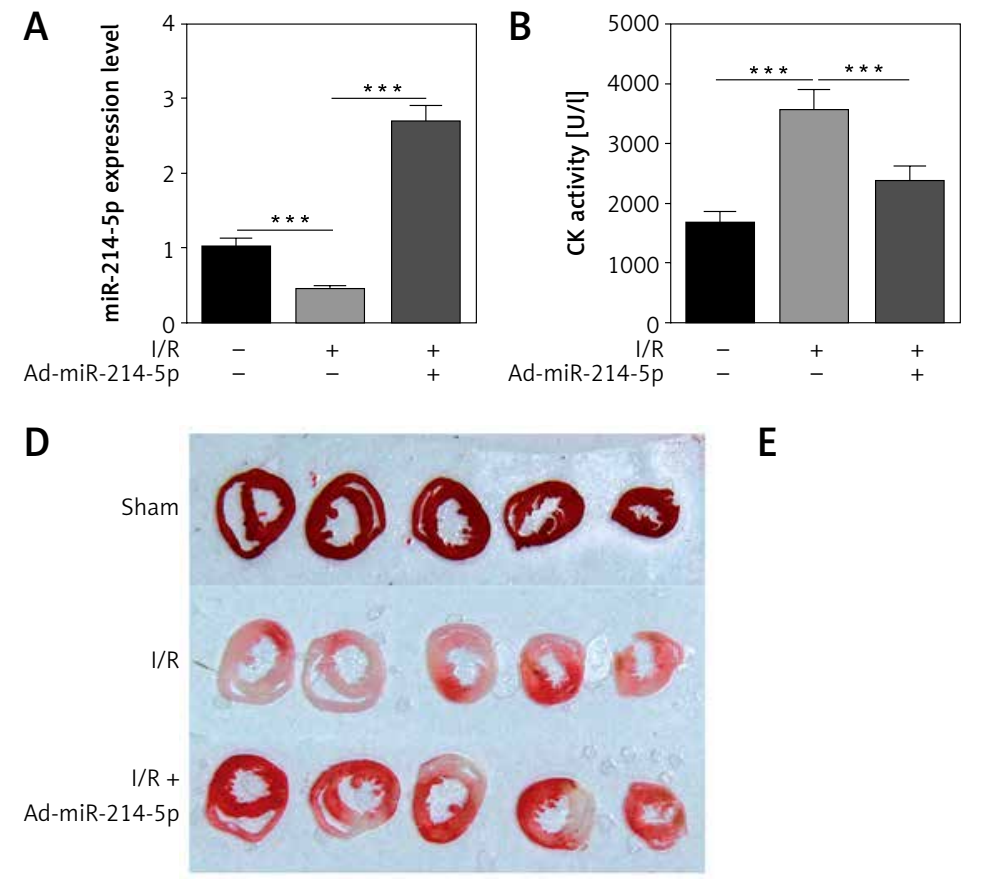

E

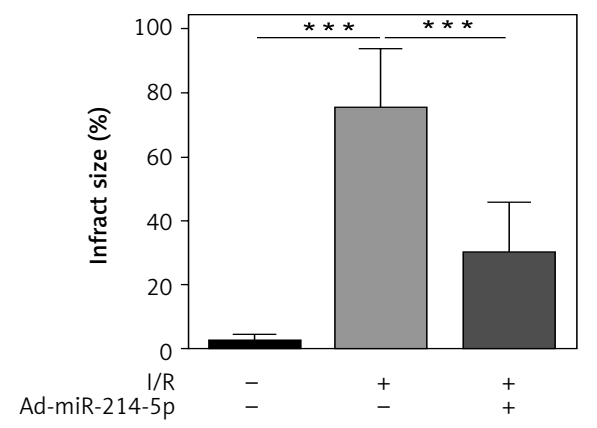

$\mathrm{F}$
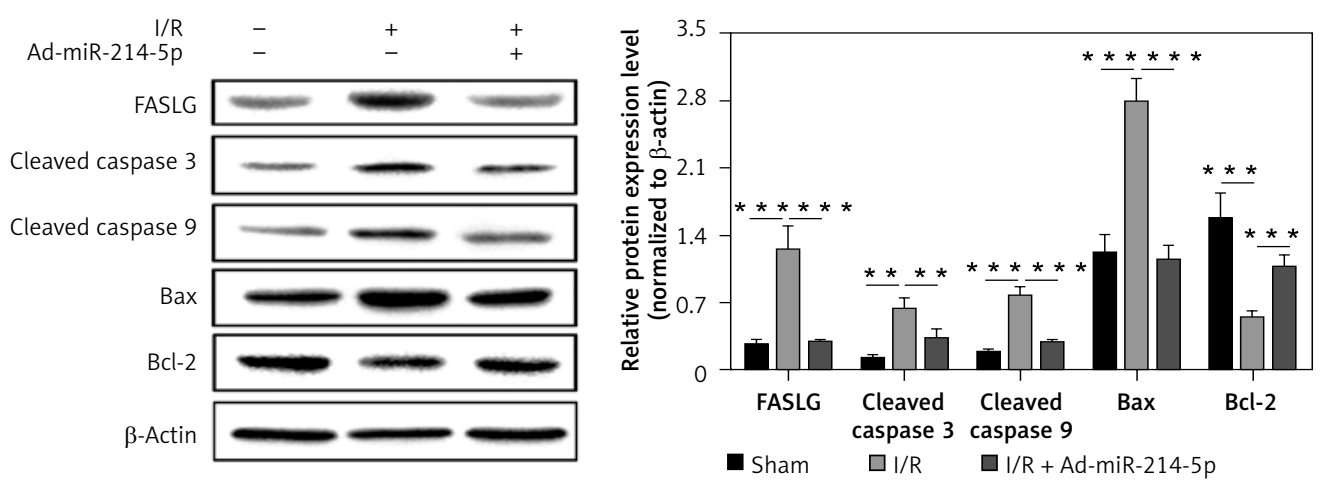

Figure 7. miR-214-5p reduced cardiac cell damage induced by I/R injury in mice. The animals were administered with Ad-miR-214-5p in an intracoronary delivery manner 5 days before the I/R operation. A - The effect of adenovirus on the expression level of miR-214-5p was evaluated by qRT-PCR in I/R-treated mice. B, C - The influence of overexpression of miR-214-5p on the CK and LDH activity was examined by CK and LDH assay kit in mice exposed to I/R treatment. D - The influence of Ad-miR-214 on the focal ischemia of heart slices and quantitative analysis of heart infarct size were assessed by TTC staining in I/R-treated animals. E - Representative images of cardiac tissue slices were acquired by HE staining in mice injected with Ad-miR-214-5p following I/R treatment. F - The effect of enhanced miR-214-5p expression on the pro- or anti-apoptotic protein levels was evaluated by western blotting in mice upon I/R exposure. $N=6$ per group

Data are expressed as mean $\pm S D ;{ }^{* *} p<0.01,{ }^{* * *} p<0.001$. 
sistently, the present study also found that FASLG and pro-apoptotic proteins Bax, cleaved-caspase 9 and 3 were up-regulated while the anti-apoptotic protein $\mathrm{BCl}-2$ was down-regulated in $\mathrm{H} / \mathrm{R}$ injured cardiac cells. Based on these results, the functions of miR-214-5p and FASLG in I/R damage have been described. Thus, the miR-214-5p/FASLG axis is considered as a potential therapy target for ischemia-induced cardiovascular diseases.

In conclusion, the current study elucidated the protective function of miR-214-5p via suppressing the FASLG-mediated apoptotic signaling pathway in $\mathrm{H} 9 \mathrm{c} 2$ cells after H/R injury. Further studies for the recovery roles of miR-214-5p in vivo will provide a post-injury treatment target of myocardial ischemia-induced disease.

\section{Acknowledgments}

This work was supported by the Youth Science Foundation program of China (Grant No. 81700231).

\section{Conflict of interest}

The authors declare no conflict of interest.

\section{References}

1. Buja LM, Vander Heide RS. Pathobiology of ischemic heart disease: past, present and future. Cardiovasc Pathol 2016; 25: 214-20.

2. Hausenloy DJ, Yellon DM. Myocardial ischemia-reperfusion injury: a neglected therapeutic target. J Clin Invest 2013; 123: 92-100.

3. Li J, Donath S, Li Y, et al. Mir-30 regulates mitochondrial fission through targeting p53 and the dynamin-related protein-1 pathway. PLoS Genet 2010; 6: e1000795.

4. Kim VN, Han J, Siomi MC. Biogenesis of small rnas in animals. Nat Rev Mol Cell Biol 2009; 10: 126-39.

5. Penna E, Orso F, Taverna D. Mir-214 as a key hub that controls cancer networks: small player, multiple functions. J Invest Dermatol 2015; 135: 960-9.

6. Zhao Y, Ponnusamy M, Zhang L, et al. The role of mir214 in cardiovascular diseases. Eur J Pharmacol 2017; 816: 138-45.

7. Wang $X, H a T$, Hu Y, et al. Microrna-214 protects against hypoxia/reoxygenation induced cell damage and myocardial ischemia/reperfusion injury via suppression of pten and bim1 expression. Oncotarget 2016; 7: 8692636.

8. Park KM, Teoh JP, Wang Y, et al. Carvedilol-responsive micrornas, mir-199a-3p and -214 protect cardiomyocytes from simulated ischemia-reperfusion injury. Am J Physiol Heart Circ Physiol 2016; 311: H371-83.

9. Rieux-Laucat F, Magerus-Chatinet A, Neven B. The autoimmune lymphoproliferative syndrome with defective fas or fas-ligand functions. J Clin Immunol 2018; 38: 558-68.

10. Salomon RN, Diaz-Cano S. Introduction to apoptosis. Diagn Mol Pathol 1995; 4: 235-38.

11. Kantari C, Walczak H. Caspase-8 and bid: caught in the act between death receptors and mitochondria. Biochim Biophys Acta 2011; 1813: 558-63.
12. Degterev A, Boyce M, Yuan J. A decade of caspases. Oncogene 2003; 22: 8543-67.

13. Fadeel B, Orrenius S. Apoptosis: a basic biological phenomenon with wide-ranging implications in human disease. J Intern Med 2005; 258: 479-517.

14. Liu XM, Yang ZM, Liu XK. Fas/fasl induces myocardial cell apoptosis in myocardial ischemia-reperfusion rat model. Eur Rev Med Pharmacol Sci 2017; 21: 2913-8.

15. Yaoita H, Ogawa K, Maehara K, et al. Apoptosis in relevant clinical situations: contribution of apoptosis in myocardial infarction. Cardiovasc Res 2000; 45: 630-41.

16. Wan DY, Zhang Z, Yang HH. Cardioprotective effect of mir-214 in myocardial ischemic postconditioning by down-regulation of hypoxia inducible factor 1, alpha subunit inhibitor. Cell Mol Biol (Noisy-le-grand) 2015; 61: 1-6.

17. Liu PY, Tian Y, Xu SY. Mediated protective effect of electroacupuncture pretreatment by mir-214 on myocardial ischemia/reperfusion injury. J Geriatr Cardiol 2014; 11: 303-10.

18. Liu H, Guo X, Chu Y, et al. Heart protective effects and mechanism of quercetin preconditioning on anti-myocardial ischemia reperfusion (ir) injuries in rats. Gene 2014; 545: 149-55.

19. Kishore Kumar G, Rajesh Kumar G, Mrudula Spurthi K, et al. Polymorphisms of extrinsic death receptor apoptotic genes (fas $-670 \mathrm{~g}>\mathrm{a}$, fasl $-844 \mathrm{t}>\mathrm{c}$ ) in coronary artery disease. Apoptosis 2016; 21: 558-65.

20. Ristic T, Djordjevic VB, Deljanin-llic M, et al. Serum fas/ fasl levels in dependence on clinical presentations of coronary disease and their relationship with risk factors. Vojnosanit Pregl 2010; 67: 537-42.

21. Lu C, Ren D, Wang X, et al. Toll-like receptor 3 plays a role in myocardial infarction and ischemia/reperfusion injury. Biochim Biophys Acta 2014; 1842: 22-31.

22. Yao X, Tan G, He C, et al. Hydrogen sulfide protects cardiomyocytes from myocardial ischemia-reperfusion injury by enhancing phosphorylation of apoptosis repressor with caspase recruitment domain. Tohoku J Exp Med 2012; 226: 275-85.

23. Elmadbouh I, Ashraf M. Tadalafil, a long acting phosphodiesterase inhibitor, promotes bone marrow stem cell survival and their homing into ischemic myocardium for cardiac repair. Physiol Rep 2017; 5: pii: e13480. 